\title{
Clinical and Functional Outcomes of Robotic Assisted Bicompartmental Knee Arthroplasty
}

\author{
Ryan Moore MD ${ }^{1}$, Thomas Coon MD ${ }^{1}$, Jennifer DeBattista BS ${ }^{1}$, Sarah Shi \\ $\mathrm{MS}^{2}$, Alexandra Valentino BS${ }^{2}$, and Manoshi Bhowmik-Stoker $\mathrm{PhD}^{2}$ \\ ${ }^{1}$ St. Helena Coon Joint Replacement Institute, St. Helena, CA, USA \\ ${ }^{2}$ Stryker, Mahwah, NJ, USA \\ rymoore2009@gmail.com, Coontm@gmail.com, DeBattJL@ah.org, \\ Sarah.Shi@stryker.com, Alexandra.Valentinolstryker.com, \\ manoshi.bhowmik-stoker@stryker.com
}

\begin{abstract}
This study reports on mid-term survivorship and outcomes of medial bicompartmental robotic assisted primary knee arthroplasty.

Forty-six knees in 43 patients with a mean 6 year follow up were consented to participate in this retrospective data collection. All patients received primary treatment for knee osteoarthritis with fixed metal backed medial femoral and patellar implants. Demographic data along with post-operative outcomes were collected including; the KSS patient portion, implant survivorship, and patient satisfaction.

All patients were included in the survivorship analysis. 45 knees in 42 patients were available for analysis of KSS and patient satisfaction. The mean follow-up was 6.1 years (range 2.1 - 7.6 years). Sixty-three percent of patients were male and $37 \%$ female. Average age at the time of surgery was 67 years and average BMI was 29. There were 3 revisions and 1 arthroscopic surgery resulting in $97.8 \%$ all cause survivorship. $86.7 \%$ of patients reported walking more than 10 blocks and $95.6 \%$ of patients reported walking without the use of assistive devices for support. All patients were able to go up and down stairs, $48.9 \%$ required use of a rail. $82.2 \%$ of patients reported being satisfied with their knee at 5 years.

In this study we found $97.8 \%$ survivorship at a mean 6 year follow up with $82.2 \%$ of patients being very satisfied or satisfied with the implant. Longitudinal follow up at 7 and 10 years will continue to evaluate long term outcomes on the variations of robotic assisted knee arthroplasty.
\end{abstract}




\section{Introduction}

Bicompartmental knee arthroplasty is indicated for patients who have end stage osteoarthritis in more than one compartment of the knee. Combined compartmental arthroplasties can be an alternative to Total Knee Arthroplasty with the advantages of preserving bone stock and restoring more normal kinematics. 1 While this procedure may be a viable alternative to Total Knee Arthroplasty, component placement continues to be challenging with conventional surgical instruments resulting in high early failure rates. 2 Recently, robotic-arm assisted Partial Knee Arthroplasty has shown to be successful in component positioning through pre-op planning and intra-op adjustability. 3 Clinical outcomes of this technology are not well established and need to be investigated. This study reports on mid-term survivorship and outcomes of medial bicompartmental robotic assisted primary knee arthroplasty.

\section{Materials and Methods}

Forty-six knees in 43 patients with a mean 6 year follow up were consented to participate in this retrospective data collection. All patients received primary treatment for knee osteoarthritis with fixed metal backed medial femoral and patellar implants. Three patients had staged bilateral bicompartmental knee arthroplasty. Demographic data along with post-operative outcomes were collected including; the KSS patient portion, implant survivorship, and patient satisfaction.

\section{Results}

All patients were included in the survivorship analysis. One patient was revised at 2 years and postoperative questionnaires were no longer collected. This resulted in 45 knees in 42 patients available for analysis of KSS and patient satisfaction. The mean follow up was 6.1 years (range 2.1 - 7.6 years). Sixty three percent of patients were male and $37 \%$ female. Average age at the time of surgery was 67 years and average BMI was 29. There were 3 revisions and 1 arthroscopic surgery resulting in $97.8 \%$ all cause survivorship [Fig 1]. 86.7\% of patients reported walking more than 10 blocks and $95.6 \%$ of patients reported walking without the use of assistive devices for support. $2.2 \%$ of patients uses a cane for walking support due to a disease unrelated to their operative knee. All patients were able to go up and down stairs, $48.9 \%$ required use of a rail. $82.2 \%$ of patients reported being very satisfied or satisfied with the function of their knee at 5 years.

\section{Discussion and Conclusion}

Bicompartmental knee arthroplasty retains the patient's ACL and can preserve native knee mechanics. In this study we found $97.8 \%$ survivorship at a mean 6 year follow up with $82.2 \%$ of patients being very satisfied or satisfied with the implant. This data is similar to the results on robotic armassisted Unicompartmental Knee Arthroplasty and Bicompartmental Knee Arthroplasty data. In one publication where the authors followed up with Unicompartmental and Bicompartmental patients at 2 years, $89.5 \%$ of patients were satisfied or very satisfied. 4 In another study that focused solely on medial Unicompartmental Knee Arthroplasty with a follow up of 5 years, the survivorship rate was $97 \%$ and $91 \%$ of patients was either satisfied or very satisfied with their knee function. 5 Longitudinal follow up 
at 7 and 10 years will continue to evaluate long term outcomes on the variations of robotic assisted knee arthroplasty.

\section{Figures and Tables}

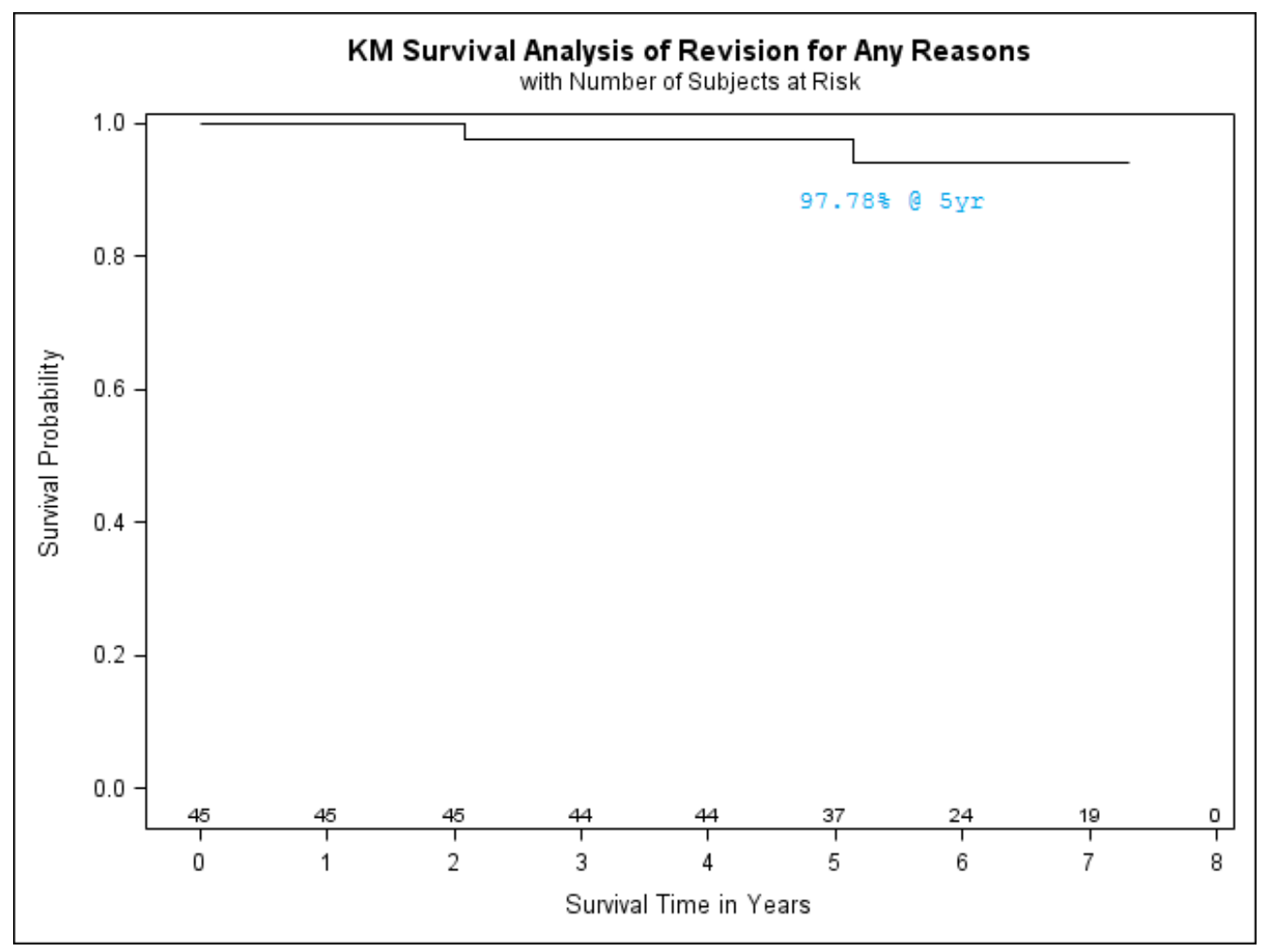

\section{References}

[1] Parratte S, Ollivier M, Lunebourg A, Abdel M.P, Argenson, J-N. Long-term results of compartmental arthroplasties of the knee. Bone Joint J 2015;97-B (10 Suppl A):9-15.

[2] Geller J, Yoon R, Macaulay, W. Unicompartmental Knee Arthroplasty. J Knee Surg. 2008; 21:714.

[3] Bell SW; Anthony I; Jones B; MacLean A; Rowe P; Blyth M. Improved accuracy of component positioning with robotic-assisted unicompartmental knee arthroplasty: data from a prospective, randomized controlled study. J Bone and Joint Surg. 2016;98: 627-35. 17.

[4] Coon T, Shi S, DeBattista J, Bhowmik-Stoker M. Clinical and functional outcomes of robotic-arm assisted unicompartmental and bicompartmental knee arthroplasty. European Knee Society 2017 Annual Meeting. London, England. Poster No. P60. April 19-21, 2017. 
[5] Kleeblad LJ, Borus T, Coon T, Dounchis J, Nguyen J, Pearle A. Midterm Survivorship and Patient Satisfaction of Robotic-Arm Assisted Medial Unicompartmental Knee Arthroplasty: A Multicenter Study. The Journal of Arthroplasty. 2018: 1-8. 